\title{
La COP21
}

La COP21 a constitué incontestablement un progrès par rapport notamment à Kyoto et Copenhague. C'est en effet la première fois que tous les pays sont d'accord pour admettre la réalité et la gravité des perspectives du changement climatique et se sont déclarés prêts à prendre des mesures pour en diminuer l'incidence. Il n'en reste pas moins vrai que les mesures proposées à ce jour par les États sont encore disparates et sans doute largement insuffisantes par rapport à l'objectif d'une hausse moyenne limitée à $2{ }^{\circ} \mathrm{C}$ et a fortiori à $1,5^{\circ} \mathrm{C}$.

Le tableau ci-après résume les forces et les faiblesses ainsi que les opportunités et les menaces à la suite de l'accord de la COP21. 
Force

Un consensus de 195 pays pour lutter contre le changement climatique par une diminution des émissions de GES Une ratification dans les délais très courts initialement prévus

Un engagement des grands États, dont les États-Unis et la Chine

Des objectifs ambitieux : limiter le réchauffement à $1,5^{\circ} \mathrm{C}$ plutôt que $2{ }^{\circ} \mathrm{C}$

Des échéances assez précises avec un calendrier rapproché

Un dispositif de révision tous les 5 ans

Un dispositif de suivi, sur une base déclarative

L'instauration de la transparence et d'un suivi mutuel entre les États

Pas de possibilité de mise en cause de la responsabilité passée des États développés

Des efforts demandés modulés selon la situation des États, prenant en compte les besoins de croissance des moins développés

Un équilibre entre actions pour réduire les émissions de GES et celles pour renforcer les capacités d'adaptation aux effets néfastes des changements climatiques

La prise en compte de la mobilisation des acteurs (collectivités locales, industries, associations, citoyens) comme force de propositions et d'initiatives

\section{Faiblesse}

Pas de mécanisme véritablement contraignant

Pas de financiarisation des externalités liées aux émissions de gaz à effet de serre; pas de taxe carbone ou autre mécanisme de régulation mondiale

Trafics aériens et maritimes non pris en compte

Contribution des pays développés aux Fonds en faveur des pays en voie de développement non finalisée : laissée au volontariat, avec une dotation plancher indicative

Pas de référence explicite aux énergies renouvelables 


\begin{tabular}{|c|c|}
\hline Opportunités & Menaces \\
\hline $\begin{array}{l}\text { La flexibilité donnée à chaque pays } \\
\text { sur les voies et moyens de réduire } \\
\text { ses productions de gaz à effet de } \\
\text { serre } \\
\text { Les opportunités de développements } \\
\text { technologiques et économiques } \\
\text { La compatibilité affirmée entre } \\
\text { croissance et réduction des émissions } \\
\text { de GES, y compris par des actions de } \\
\text { captures et de stockage, permettant } \\
\text { la poursuite de l'usage d'énergies } \\
\text { fossiles durant un certain temps } \\
\text { La place faite aux initiatives non } \\
\text { gouvernementales, propice à la } \\
\text { mobilisation collective }\end{array}$ & $\begin{array}{l}\text { Une remise en cause des } \\
\text { engagements de certains États, } \\
\text { à commencer par les États- } \\
\text { Unis, du fait d'alternances } \\
\text { gouvernementales } \\
\text { L'insuffisance des objectifs } \\
\text { nationaux cumulés } \\
\text { L'insuffisance des politiques } \\
\text { nationales mises en œuvre } \\
\text { La non-fiabilité des données de } \\
\text { suivi des émissions } \\
\text { La priorité donnée à la croissance } \\
\text { par rapport à la réduction des } \\
\text { émissions de GES, notamment } \\
\text { dans les pays en développement } \\
\text { L'incapacité mondiale à financer la } \\
\text { transition }\end{array}$ \\
\hline
\end{tabular}


